\title{
FREUD'S CONJECTURE FOR EXPONENTIAL WEIGHTS
}

\author{
D. S. LUBINSKY, H. N. MHASKAR AND E. B. SAFF
}

1. Freud's Conjecture. Let $W(x)$ be nonnegative on $\mathbf{R}$, positive on a set of positive Lebesgue measure, and let $W^{2}(x)$ have all moments finite. Then we call $W$ a weight function. Associated with $W^{2}(x)$ are the orthonormal polynomials

$$
p_{n}\left(W^{2} ; x\right):=\gamma_{n}\left(W^{2}\right) x^{n}+\cdots, \quad n=0,1,2,3, \ldots,
$$

satisfying

$$
\int_{-\infty}^{\infty} p_{m}\left(W^{2} ; x\right) p_{n}\left(W^{2} ; x\right) W^{2}(x) d x=\delta_{m n} .
$$

While asymptotics for the ratio $\gamma_{n-1} / \gamma_{n}, n \rightarrow \infty$, are classical for weights on $[-1,1]$, only recently have analogous results been considered for the more difficult problem of weights on $\mathbf{R}$. In 1974, G. Freud [2] conjectured that if $W(x)=W_{\alpha, \rho}(x)$, where

$$
W_{\alpha, \rho}(x):=|x|^{\rho / 2} \exp \left(-|x|^{\alpha}\right), \quad x \in \mathbf{R}, \alpha>0, \rho>-1,
$$

then

$$
\lim _{n \rightarrow \infty} n^{-1 / \alpha} \gamma_{n-1}\left(W_{\alpha, \rho}^{2}\right) / \gamma_{n}\left(W_{\alpha, \rho}^{2}\right)
$$

exists. He expressed the value that the limit should take in terms of gamma functions, and proved his conjecture for $\alpha=2,4,6$. Recently, Al. Magnus [8] proved the conjecture for $\rho>-1$ and $\alpha$ a positive even integer, and subsequently [9] for weights of the form $\exp (-P(x))$, where $P(x)$ is a polynomial of even degree with positive leading coefficient. Máté, Nevai, and Zaslavsky [11] have sharpened Magnus' result to an asymptotic expansion. Several applications of Freud's Conjecture are discussed by Nevai [16], and related physical applications have been considered by Bessis, Itzykson, and Zuber [1] and in $[\mathbf{1 7}]$.

The purpose of this paper is to announce a proof of Freud's Conjecture for a general class of weights that includes $W_{\alpha, \rho}(x)$ for all $\alpha>0, \rho>-1$. In describing the analogue of the conjecture for general weights, a crucial role is played by the number $a_{n}=a_{n}(W)$, introduced by Mhaskar and Saff in $[\mathbf{1 3}, 14]$. Let $W(x)=\exp (-Q(x))$, where $Q(x)$ is even, continuous in $\mathbf{R}$, and

Received by the editors May 15, 1986.

1980 Mathematics Subject Classification (1985 Revision). Primary 42C05; Secondary 33A65, 41A10.

Research of the first author was completed while the author was visiting the Institute for Constructive Mathematics, Department of Mathematics, University of South Florida.

The second author is grateful to the California State University, Los Angeles for a leave of absence during which this work was performed.

Research of the third author supported, in part, by the National Science Foundation. 
differentiable for $x>0$, while $x Q^{\prime}(x)$ remains bounded as $x \rightarrow 0$. Whenever uniquely defined, $a_{n}=a_{n}(W)$ is taken to be the positive root of the equation

$$
n=\frac{2}{\pi} \int_{0}^{1} \frac{a_{n} x Q^{\prime}\left(a_{n} x\right) d x}{\sqrt{1-x^{2}}}
$$

Under certain conditions on $Q$, it is shown in [14] that for every polynomial $P(x)$ of degree at most $n$,

$$
\|P W\|_{L_{\infty}(\mathbf{R})}=\|P W\|_{L_{\infty}\left[-a_{n}, a_{n}\right]}
$$

and that $a_{n}$ is asymptotically best possible.

The set of weights for which we can prove Freud's Conjecture includes the following class.

DEFINITION 1.1. Let $W(x)=\exp (-Q(x))$, where $Q(x)$ is even and continuous and $Q^{\prime \prime \prime}(x)$ exists for $x>0$, while $x Q^{\prime}(x)$ is bounded as $x \rightarrow 0+$, and for some $C>0$ and $\alpha>0$,

$$
\begin{gathered}
Q^{\prime}(x)>0, \quad x \text { large enough, } \\
x^{2}\left|Q^{\prime \prime \prime}(x)\right| / Q^{\prime}(x) \leq C, \quad x \text { large enough, }
\end{gathered}
$$

and

$$
\lim _{x \rightarrow \infty}\left(1+x Q^{\prime \prime}(x) / Q^{\prime}(x)\right)=\alpha .
$$

Then we shall call $W$ a very smooth Freud weight of order $\alpha$ and write $W \in$ $\operatorname{VSF}(\alpha)$.

The conditions on $Q$ may be slightly weakened, but we retain the above formulation for simplicity. These conditions arise in the construction of an even entire function with nonnegative Maclaurin series coefficients that behaves like $W^{-1}(x)$ on $\mathbf{R}$ (Lubinsky $[\mathbf{4}, \mathbf{5}]$ ), and in ensuring that the zero distribution of extremal polynomials associated with $W$ behaves asymptotically like the Ullman distribution $[6,14]$. In particular, if $Q(x)=|x|^{\alpha}\left(\log \left(2+x^{2}\right)\right)^{\beta}, \alpha>$ $0, \beta \in \mathbf{R}$, then $W(x)=\exp (-Q(x)) \in \operatorname{VSF}(\alpha)$. We note that if $W \in \operatorname{VSF}(\alpha)$, then $a_{n}$ is uniquely defined by (1.1) for $n$ large enough.

THEOREM 1.2. Let $W \in \operatorname{VSF}(\alpha)$ for some $\alpha>0$ and let $\rho>-1$. If $a_{n}:=$ $a_{n}(W)$ is defined by (1.1) and $\gamma_{n}:=\gamma_{n}\left(|x|^{\rho} W^{2}\right)$ are the leading coefficients of the orthonormal polynomials associated with $|x|^{\rho} W^{2}$, then

$$
\lim _{n \rightarrow \infty} a_{n}^{-1} \gamma_{n-1} / \gamma_{n}=1 / 2
$$

In the special case $W(x)=\exp \left(-|x|^{\alpha}\right), \alpha>0,(1.6)$ may be rewritten in the form

$$
\lim _{n \rightarrow \infty} n^{-1 / \alpha} \gamma_{n-1}\left(|x|^{\rho} W_{\alpha}^{2}\right) / \gamma_{n}\left(|x|^{\rho} W_{\alpha}^{2}\right)=\beta_{\alpha} / 2
$$

where

$$
\beta_{\alpha}:=\left\{\frac{\Gamma(\alpha)}{2^{\alpha-2} \Gamma(\alpha / 2)^{2}}\right\}^{-1 / \alpha} .
$$


We shall also prove in [7] the analogue of Freud's Conjecture for noneven weights such as $|x|^{\rho} W^{2} e^{-P}$, where $\rho>-1, W \in \operatorname{VSF}(\alpha)$ for some $\alpha>0$, and $P$ is a polynomial of degree less than $\alpha$.

NOTE ADDED IN PROOF. Asymptotics for $\gamma_{n}(W)$ and its $L_{p}$ analogue $(1 \leq p \leq \infty)$, rather than just $\gamma_{n-1}(W) / \gamma_{n}(W)$, have also been obtained for weights such as $\exp \left(-|x|^{\alpha}\right), \alpha>0$, but for a less general class than in Theorem 1.2.

2. Outline of the proof. We shall briefly discuss the main threads of the proof of Theorem 1.2. Note first that one equivalent formulation of (1.2) is

$$
\left\|P_{n}(x) W\left(a_{n} x\right)\right\|_{L_{\infty}(\mathbf{R})}=\left\|P_{n}(x) W\left(a_{n} x\right)\right\|_{L_{\infty}[-1,1]},
$$

for each polynomial $P_{n}(x)$ of degree at most $n$. Under mild conditions on $W$, results in $[\mathbf{1 3}, \mathbf{1 4}]$ imply that any sequence of weighted polynomials $\left\{P_{n}(x) W\left(a_{n} x\right)\right\}_{n=1}^{\infty}$ uniformly bounded in $\mathbf{R}$ will converge to zero outside $[-1,1]$. Concerning the behavior of such sequences in $[-1,1]$, the following result is proved in Lubinsky and Saff [6].

THEOREM 2.1. Let $W \in \operatorname{VSF}(\alpha)$ for some $\alpha>0$ and $a_{n}:=a_{n}(W)$ be defined by (1.1). Let $g \in L_{p}(\mathbf{R}), 0<p<\infty$. Then there exist polynomials $P_{n}(x)$ of respective degrees at most $n, n=1,2,3, \ldots$, such that

$$
\lim _{n \rightarrow \infty}\left\|g(x)-P_{n}(x) W\left(a_{n} x\right)\right\|_{L_{p}(\mathbf{R})}=0
$$

if and only if $g(x)=0$ for almost all $|x| \geq 1$.

There is also an $L_{\infty}$ analogue of Theorem 2.1 which appears in [6]. One corollary to Theorem 2.1 is the solution to an $L_{p}$ analogue of a problem of Saff $[18$, p. 252], concerning approximation by weighted polynomials of the form $P_{n}(x) \exp \left(-n|x|^{\alpha}\right)$ :

COROllaRY 2.2. Let $\alpha>0$. Let $0<p<\infty$ and $g \in L_{p}(\mathbf{R})$. Then there exist polynomials $P_{n}(x)$ of respective degrees at most $n, n=1,2,3, \ldots$, such that

$$
\lim _{n \rightarrow \infty}\left\|g(x)-\exp \left(-n|x|^{\alpha}\right) P_{n}(x)\right\|_{L_{p}(\mathbf{R})}=0,
$$

if and only if $g(x)=0$ for almost all $|x| \geq \beta_{\alpha}$.

A second ingredient in the proof of Theorem 1.2 is the following sufficient condition for Freud's Conjecture for even weights [3]. An analogue for noneven weights will also appear in $[\boldsymbol{3}]$. Its proof relies entirely on methods and results developed by Máté, Nevai, and Totik [10] in their proof of Rakhmanov's theorem and extensions thereof.

THEOREM 2.3. Let $W$ be a weight function. Suppose there exists an increasing sequence of positive numbers $\left\{c_{n}\right\}_{n=1}^{\infty}$ and a decreasing sequence of positive numbers $\left\{\delta_{n}\right\}_{n=1}^{\infty}$ with

$$
\lim _{n \rightarrow \infty} \delta_{n}=0
$$


such that for $n=1,2,3, \ldots$, and every polynomial $P(x)$ of degree at most $n$,

$$
\|P W\|_{L_{2}(\mathbf{R})} \leq\left(1+\delta_{n}\right)\|P W\|_{L_{2}\left[-c_{n}, c_{n}\right]} .
$$

Further, suppose there exist polynomials $S_{n}(x)$ of respective degrees at most $n-2, n=2,3, \ldots$, such that for $p=1 / 2$ and $p=2$,

$$
\lim _{n \rightarrow \infty} \frac{1}{\pi} \int_{-1}^{1}\left|S_{n}(x) W\left(c_{n} x\right)\left(1-x^{2}\right)^{1 / 4}\right|^{p} \frac{d x}{\sqrt{1-x^{2}}}=1
$$

Then

$$
\lim _{n \rightarrow \infty} c_{n}^{-1} \gamma_{n-1}\left(W^{2}\right) / \gamma_{n}\left(W^{2}\right)=1 / 2 .
$$

It is not difficult to see that if $c_{n}$ in (2.5) is replaced by $a_{n}$, then (2.5) is an essentially weaker form of (2.2) with $p=\infty$. The final ingredient in the proof is to establish $L_{2}$ inequalities like those in (2.4), with $c_{n}$ replaced by $a_{n}\left(1+\varepsilon_{n}\right)$, where $\left\{\varepsilon_{n}\right\}_{n=1}^{\infty}$ is a sequence of positive numbers with

$$
\lim _{n \rightarrow \infty} \varepsilon_{n}=0 \text {. }
$$

Although such inequalities are implicit in results of Mhaskar and Saff $[\mathbf{1 2}, \mathbf{1 4}$, 15], they have not been formally stated in the literature. One consequence of results of Mhaskar and Saff $[\mathbf{1 2}, \mathbf{1 5}]$ is the following:

THEOREM 2.4. Let $W \in \operatorname{VSF}(\alpha)$ for some $\alpha>0$. Let $a_{n}:=a_{n}(W)$ be defined by (1.1) for $n$ large enough. Let $0<p \leq \infty$ and $\delta>0$. Then there exists $C_{1}, C_{2}>0$ such that for $n=1,2,3, \ldots$, and all polynomials $P$ of degree at most $n$,

$$
\|P(x) W(x)\|_{L_{p}(\mathbf{R})} \leq\left(1+C_{1} e^{-C_{2} n}\right)\|P(x) W(x)\|_{L_{p}\left(-a_{n}(1+\delta), a_{n}(1+\delta)\right)} .
$$

This is stated and proved in [6]. The precise inequalities required for application of Theorem 2.3, as well as full details of the proof of Theorem 1.2, will appear in [7].

\section{REFERENCES}

1. D. Bessis, C. Itzykson, and J. B. Zuber, Quantum field theory techniques in graphical enumeration, Adv. in Appl. Math. 1 (1980), 109-157.

2. G. Freud, On the coefficients in the recursion formulae of orthogonal polynomials, Proc. Royal Irish Acad. Sect. A 76 (1976), 1-6.

3. A. Knopfmacher, D. S. Lubinsky, and P. Nevai, Freud's conjecture and approximation of reciprocals of weights by polynomials (manuscript).

4. D. S. Lubinsky, Gaussian quadrature, weights on the whole real line and even entire functions with nonnegative even order derivatives, J. Approximation Theory (to appear).

5. Even entire functions absolutely monotone in $[0, \infty)$ and weights on the whole real line, Orthogonal Polynomials and Their Applications (C. Brezinski et al., eds.), Lecture Notes in Math., Springer-Verlag, Berlin and New York, 1986.

6. D. S. Lubinsky and E. B. Saff, Uniform and mean approximation by certain weighted polynomials, with applications (manuscript).

7. D. S. Lubinsky, H. N. Mhaskar, and E. B. Saff, A proof of Freud's Conjecture for exponential weights (manuscript). 
8. Al. Magnus, A proof of Freud's Conjecture about orthogonal polynomials related to $|x|^{\rho} \exp \left(-x^{2 m}\right)$, Orthogonal Polynomials and Their Applications (C. Brezinski et al., eds.) Lecture Notes in Math., Springer-Verlag, Berlin and New York, 1986.

9. __ On Freud's equations for exponential weights, J. Approximation Theory 46 (1986), 65-99.

10. A. Máté, P. Nevai, and V. Totik, Asymptotics for the ratio of leading coefficients of orthonormal polynomials on the unit circle, Constr. Approx. 1 (1985), 63-69.

11. A. Máté, P. Nevai, and T. Zaslavsky, Asymptotic expansion of ratios of coefficients of orthonormal polynomials with exponential weights, Trans. Amer. Math. Soc. 287 (1985), 495-505.

12. H. N. Mhaskar and E. B. Saff, Extremal problems for polynomials with exponential weights, Trans. Amer. Math. Soc. 285 (1984), 203-234.

13. Weighted polynomials on finite and infinite intervals: A unified approach, Bull. Amer. Math. Soc. (N.S.) 11 (1984), 351-354.

14. - Where does the sup norm of a weighted polynomial live? (A generalization of incomplete polynomials), Constr. Approx. 1 (1985), 71-91.

15. _ Where does the $L^{p}$ norm of a weighted polynomial live? (manuscript).

16. P. Nevai, Geza Freud, Christoffel functions and orthogonal polynomials ( $A$ case $s t u d y$ ), J. Approximation Theory (to appear).

17. D. G. Pettifor and D. L. Weaire (eds.), The recursion method and its applications, Springer Series in Solid State Physics, vol. 58, Springer-Verlag, Berlin and New York, 1984.

18. E. B. Saff, Incomplete and orthogonal polynomials, Approximation Theory IV (C. K. Chui et al., eds.), Academic Press, New York, 1983, pp. 219-256.

NATIONAl REsearch InStitute For Mathematical SCIENCES, C.S.I.R., P.O. BoX 395, Pretoria 0001, Republic of South Africa

Department of MATHEMATiCs ANd Computer SCIENCE, California State UNIVERSity, LOS ANGEles, CALIFORNiA 90032

Institute for Constructive MATHEMATiCs, Department of MATHEMATICS, UNIVERSITY OF SOUTH FLORIDA, TAMPA, FLORIDA 33620 